\section{Mycobacterium tuberculosis complex: Detection and patterns of resistance to the first line anti-TB drugs at the King Abdulaziz University Hospital, Saudi Arabia}

\section{Abstract}

Background: Tuberculosis (TB) remains a major cause of morbidity and mortality worldwide. Continued surveillance of drug susceptibility helps determining proper treatment regimen. This study aimed to investigate occurrence, patterns of anti-tuberculosis drug-susceptibility at the King Abdulaziz University Hospital (KAUH), Jeddah, KSA.

Methods: This study represents a cross sectional study and including a total of 101 TB patients examined in the Clinical and Molecular Microbiology Laboratory at the KAUH from January 2013 to June 2014. They were 43 Saudi and 58 non-Saudi patients. Each specimen was digested, decontaminated, and its pellet was used for preparation fluorochrome stain. The rest of each pellet was used for culture in the VersaTREK liquid culture system as well as used for direct MTBC detection by GeneXpert PCR. The susceptibility to anti-TB drugs was done by VersaTREK.

Results: The sensitivity of PCR for MTBC detection was $88.1 \%$ for AFB-positive specimens and $29.9 \%$ for AFB-negative ones, while the sensitivity of PCT for MTBC detection was $74.3 \%$ and $51.9 \%$ for pulmonary and extra-pulmonary specimens, respectively. VersaTREK detection time was $15.01 \pm 7.32$ and $26.63 \pm 6.7$ days for positive and negative AFB specimens, respectively. The resistance rate to pyrazinamide was (13.86\%), followed by streptomycin (7.9\%), rifampicin (3.96\%) and isoniazid (3.96\%). Mono-resistance rates to pyrazinamide, rifampicin
Asif Jiman-Fatani ${ }^{1,3}$, Dalia El-Hossary2,3 and Rehab A. Eltahlawi ${ }^{2}$

1 Department of Medical Microbiology and Parasitology, Faculty of Medicine, King Abdulaziz University, Jeddah, Kingdom of Saudi Arabia.

2 Medical Microbiology and Immunology Department, Faculty of Medicine, Zagazig University, Egypt.

3 Clinical and Molecular Microbiology Laboratory, King Abdulaziz University Hospital, Jeddah, Kingdom of Saudi Arabia.

Corresponding author:

Dr. Rehab A. Eltahlawi

” rehabt6@gmail.com 
and isoniazid were $11.88 \%, 1.98 \%$ and $0.99 \%$, respectively. MDR-TB were $1.98 \%$, and there was no significant difference between Saudi and non-Saudi TB patients regarding the resistance to anti-TB drugs.

Conclusion: Further studies and continued surveillance of the drug resistance patterns of $M$. tuberculosis are needed to reduce risk factors of infection with MDR-TB, and to formulate effective chemotherapy in the management of TB cases.

Key words: Mycobacteria Detection, Anti-TB drugs, Saudi Arabia

\section{Introduction}

Tuberculosis (TB) remains a major cause of morbidity and mortality worldwide. The World Health Organization(WHO) estimates that onethird of the population of the world is infected with Mycobacterium tuberculosis and that more than 8 million new cases of active TB occur annually [1]. Today, there are two monumental threats to global TB control; the HIV epidemic and the increasing prevalence of anti-tuberculosis drug-resistance [2].

There are important factors contribute to emergence of anti-tuberculosis drug-resistance, including treatment with a single drug, inappropriate prescription or poor adherence to treatment, suppression the growth of susceptible bacilli to used drug, while permits the development of drugresistant bacilli. Subsequent transmission of resistant TB bacilli from an infectious case to other susceptible persons may lead to TB primary resistance $[3,4]$.

The Kingdom of Saudi Arabia (KSA) is among the most dynamic immigration hubs on the globe. It hosts approximately 8.4 to 10 million expatriates and annually receives another 10 million for Islamic rituals (Hajj and Umrah) compared to a population of 18.7 million nationals. The majority of the visitors and expatriates are coming from countries with high incidence of TB such as South-East Asia, Indian subcontinent and Africa. Many of these foreign-born residents and pilgrims reside at least for one-week or much longer, in overcrowded and suboptimal living conditions, and in constant and close contact with Saudi citizens. These factors enhance TB transmission among the country population [5].

In KSA, the pattern of anti-TB drug resistance showed marked regional variation. Analysis of local rates of drug-resistance provides the necessity of using the four-drug regimen consisting of isoniazid (INH), rifampicin (RIF), ethambutol (EMB) and pyrazinamide (PZA). Therefore, continued surveillance of drugs susceptibility patterns is necessary to determine the extent and patterns of anti-TB drug resistance in KSA[6]. The solid mediumbased drug-susceptibility testing (DST) method is slow and delays the detection of drug-resistance M. tuberculosis, increased risks of inappropriate treatment and spread of drug-resistant strains. These factors have led to the development of newer DST methodologies, includinguse of liquid culture systems according to recent recommendations of WHO [7]. Additionally, several direct techniques for DNA amplification of $M$. tuberculosisfrom in sputum have received approval by the US Food and Drug Administration (FDA). However, most of these rapid tests, do not provide full information about anti-TB drug-susceptibility [8]. 
The present study aims to investigate the role of PCR for the rapid detection of MTBC directly in clinical samples as compared to the liquid culture (VersaTREK), and to determine the pattern of antiTB drug-resistance at the King Abdulaziz University Hospital, Jeddah.

\section{Materials and Methods}

This prospective cross-sectional study was done in the Clinical and Molecular Microbiology Laboratory at the King Abdulaziz University Hospital (KAUH), Jeddah, KSA from January 2013 through June 2014.

\section{Ethical consideration}

The study was approval by Research Ethics Committee, and the Unit of Biomedical Ethics, Faculty of Medicine, King Abdulaziz University (Reference Number: 241-15).

This study was included 101 patients diagnosed with TB. All patients were subjected to full history taking, full clinical examinations with radiological and routine laboratory investigations.

\section{Preparation of Specimens}

Each specimen was digested and decontaminated by ready to use $\mathrm{N}$-acetyl-I-cysteine (NALC) 3\% $\mathrm{NaOH}$ method (Nac-PACTMEA3, Alpha Tec, Inc, Vancouver, Washington, USA) as described by the manufacturer [7]. Part of the obtained pellet of each specimen was used for detection of M.tuberculosis Complex (MBTC) by preparing a smear using fluorochrome stain (Fluo-RAL-Auramine staining kit for Mycobacteria Detection (RAL-automated staining system-R.A.L. instruments, Montesquieu-33650 MARTILLAC-France), and later each smear was examined with the fluorescent microscope. The rest of the pellet was used for culture in the VersaTREK liquid culture system as well as for direct
MTBC detection by GeneXpert PCR (Cepheid 904 Caribbean Drive Sunnyvale, CA 94089-1189-USA).

The GeneXpert Dx System (Cepheid 904 Caribbean Drive Sunnyvale, CA 940891189-USA)

The GeneXpert Dx System integrates and automates sample processing, nucleic acid amplification, and detection of the target sequences of simple or complex samples using real time PCR and reverse transcriptase PCR.

Molecular detection of MTBC and rpoB gene mutations associated with rifampicin-resistance speeds the detection of MDR-TB. The Xpert MTB/ RIF assay can be used on fresh samples as well as on prepared sediments [8].

a) Sample sediment procedure: Each MTB/RIF cartridge was labeled by the sample ID, then $1 \mathrm{ml}$ of the total re-suspended pellet was transferred to a conical screw-capped tube for the Xpert MTB/RIF by a pipette, followed by a transfer of $2 \mathrm{ml}$ of the Xpert Sample Reagent (SR) to the re-suspended sediment, then vortex for 10 seconds was done.The conical screwcapped tube was incubated for 10 minutes at room temperature, then vortex was done and re-incubated for additional 5 minutes.The liquefied sample was transferred to the sample chamber of the Xpert MTB/ RIF cartridge and the cartridge was loaded into the GeneXpert Dx Instrument. The test was started and the running software was GX 4.0 [8].

b) Sample preparation and extraction of nucleic acids: The GeneXpert System completely integrates and automates the sample preparation and performs all the complex steps of nucleic acid extraction in its advanced microfluidic cartridge [8].

c) Amplification of the extracted nucleic acids: The GeneXpert System modules performed rapid heating and cooling cycles required for highly reliable real-time $P C R$ in the reaction tube of the cartridge [8].

d) Detection of a target gene sequence: The GeneXpert System's multiple optics can detect the 
presence of multiple target nucleic acids in the same cartridge [8].

e) Interpretation of Results: The GeneXpert System generated the results from measured fluorescent signals and embedded calculation algorithms. The detection of MTBC (very low, low, medium, or high) was dependent on the number of the TB bacilli in the sample [8]

\section{Inoculation of VersaTREK528 (Trek}

Diagnostic Systems, Inc. Westlake, Ohio, USA)

Bottle Preparation and Inoculation (Remel $^{\mathrm{TM}}$ Oxoid ${ }^{\mathrm{TM}}$ VersaTREK and Sensititre ${ }^{\mathrm{TM}}$ products) was done as recommended in MB/BacT Detection system manual; http://www.biomerieux.com [9].

\section{Classification of positive isolates by The Gene Xpert Dx system (Cepheid 904} Caribbean Drive Sunnyvale, CA 940891189-USA)

A total of $1 \mathrm{ml}$ of each positive culture bottle was used for detection of MTBC and a rpoB gene mutations associated with RIF resistance.

Susceptibility testing for Anti-TB drugs by VersaTREK (Remel'TM OxoidTM VersaTREK and Sensititre ${ }^{\mathrm{TM}}$ products)

a) Preparation of anti-tuberculous drugs: Rifampicin, isoniazid, ethambutol, streptomycin and pyrazinamide were reconstituted according to manufacturer's instructions. A total of $1.0 \mathrm{ml}$ of the positive culture was taken and $9.0 \mathrm{ml}$ of distilled water was added and vortex was done, then 0.5 $\mathrm{ml}$ of this diluted positive culture was used for inoculation of each mycobroth bottles.

\section{b) Sensitivity by Myco-Susceptibility kit:}

Eight new Mycobroth bottles were prepared for each positive MBTC culture according to manufacturer's instructions [9].

c) Sensitivity for Myco-Pyrazinamide (PZA) Kit: Two Myco broth bottles were prepared according to manufacturer's instructions [9]. d) Results Interpretation: The isolate in a drug-containing bottle is considered to be resistant when its time for detection is equal to, or within three days from the detection time of the drug-free control bottle [9].

\section{Statistical analysis}

Data were analyzed using Statistical Package for Social Sciences (SPSS) software, version 18. Chi square test was utilized to test for the association and/or difference between categorical variables. Fisher exact test was used when appropriate. Continuous variables were presented as mean, standard deviation and range. Student t test was used to compare between two groups. P value less than 0.05 was considered statistically significant.

\section{Results}

The present study includes a total of 101 confirmed infected patients with TB. They were $43(42.5 \%)$ Saudis, their ages ranged from 14 to 39 years with a mean \pm SD $(23.37 \pm 14.31$ years), and 26 (60.4\%) of them were males and 17(39.6\%) were females. The second group of the TB patients were 58 (57.5 \%) non-Saudi, their ages ranged from 1 to 92 years with a mean \pm SD $(40.3 \pm 12.6$ years), and $18(48.3 \%)$ of them were males and $40(51.7 \%)$ were females. Clinical samples were collected from various clinical units of KAUH (Table 1).

The sensitivity of GeneXpert PCR for the detection of MTBC in sputum, tracheal aspirate, bronchial wash, CSF, cyst, tissue, gastric aspirate and peritoneal fluid were $81.1 \%, 40 \%, 72.7 \%$, $60 \%, 85.7 \%, 36.4 \%, 0.0 \%$ and $50 \%$ respectively (Table 2).

The sensitivity of Gene Xpert PCR for the detection of MTBC was $29.4 \%$ in AFB-negative and $88.1 \%$ in AFB-positive samples while, the sensitivity 
Table 1. Demographic characteristics of 101 patients*

\begin{tabular}{|c|c|c|c|c|}
\hline Parameters & No. (\%) Saudi & No. (\%) Non-Saudi & Test of significance & P-value \\
\hline $\begin{array}{l}\text { Age } \\
\text { range }\end{array}$ & $14-39$ & $1-92$ & \multirow[t]{2}{*}{$t=.99$} & \multirow[t]{2}{*}{0.955} \\
\hline Mean \pm SD & $23.37 \pm 14.31$ & $40.3 \pm 12.6$ & & \\
\hline $\begin{array}{l}\text { Sex } \\
\text { Males } \\
\text { Females }\end{array}$ & $\begin{array}{l}26(60.4) \\
17(39.6)\end{array}$ & $\begin{array}{c}18(48.3) \\
40(51.7)\end{array}$ & $X^{2}=1.45$ & 0.235 \\
\hline $\begin{array}{l}\text { Hospital Ward } \\
\text { Isolation unit } \\
\text { Other units }\end{array}$ & $\begin{array}{l}22(51.2) \\
21(48.8)\end{array}$ & $\begin{array}{l}31(53.4) \\
27(46.6)\end{array}$ & $X^{2}=0.05$ & 0.48 \\
\hline
\end{tabular}

*No of Saudi patients (43) and non-Saudi patients (58).

were $80 \%, 87.5 \%, 77.8 \%$ and $100 \%$ in $A F B+1,+2,+3$ and +4 positive samples, respectively (Table 3 ).

The sensitivity of GeneXpert PCR for the detection of MTBC in all AFB-negative and positive pulmonary and extra-pulmonary specimens are shown in

\section{Table 3.}

The detection time of MTBC by VersaTREK was shorter in AFB-positive than in AFB-negative samples (15.01 \pm 7.32 vs $26.63 \pm 6.7$ days) (Table 4). Moreover, the detection time of AFB-positive was comparable in both pulmonary and extra-pulmonary specimens (Table 4).

All anti-TB drug resistance detected in our study was primary resistance (resistance among newly diagnosed cases with no previous history of treatment with anti-TB drugs or treatment for less than one month.

The resistance to pyrazinamide was the most common type of resistance (13.86\%), followed by the resistance to streptomycin (7.9\%), rifampicin (3.96\%) and $\mathrm{INH}$ (3.96\%). Monoresistance to pyrazinamide, rifampicin and $\mathrm{INH}$ were $11.88 \%, 1.98 \%$ and $0.99 \%$, respectively. Meanwhile, MDR-TB accounted to $1.98 \%$, and the rate of any drug resistance was $24.75 \%$. There was no significant difference between Saudi and nonSaudi TB patients regarding any type of resistance to anti-tuberculous drugs (Table 5).

\section{Discussion}

The worldwide increase in the rates of multidrugresistant (MDR) tuberculosis (resistance to at least rifampicin and isoniazid) has made the timely identification of resistant MTBC strains an important emergence to achieve effective disease management and to prevent their spread in community. INH and RIF are the most important first-line anti-tuberculosis drugs, and resistance to these drugs are often associated with treatment failure and fatal clinical outcome [10].

This study found no significant differences between Saudis and non-Saudi TB patients regarding the age, sex, incidence or hospitalization in different unints (Table 1). This observation was not compatible with results obtained by Abouzeid et al.[11] in their 10-year retrospective study, where non-Saudis had nearly twice the TB incidence rate compared to Saudis. Our result may be explained by the size of our patients' sample which was small to reveal a statistically significant data.

This study shows that the rate of male patients was higher than that of females (60.4\% vs. $39.6 \%$ ) among Saudi patients with no significant difference. This result might be due to the higher occupational exposure of males to droplet infections together with some other factors such as smoking. Also, 
Table 2. AFB staining results and MTBC detection by Gene Xpert in each specimen.

\begin{tabular}{|c|c|c|c|c|}
\hline \multirow{2}{*}{$\begin{array}{l}\text { Types (No. ) of specimens } \\
\text { examined }\end{array}$} & \multirow{2}{*}{\multicolumn{2}{|c|}{ No. (\%) AFB staining results }} & \multicolumn{2}{|c|}{ Detection of MTBC by PCR } \\
\hline & & & $\begin{array}{l}\text { Detected } \\
\text { No.69(\%) }\end{array}$ & $\begin{array}{c}\text { Not detected } \\
\text { No.32 (\%) }\end{array}$ \\
\hline \multirow[t]{7}{*}{ Sputum (53) } & Total sputum & $53(100)$ & 43(81.1) & 10(18.9) \\
\hline & AFB -ve & $9(17)$ & $4(44.4)$ & $5(55.6$ \\
\hline & $A F B+v e$ & $44(83)$ & 39(88.6) & $5(11.4)$ \\
\hline & +1 & 10(18.9) & $7(70)$ & $3(30)$ \\
\hline & +2 & $5(9.4)$ & $5(100)$ & $0(0.0)$ \\
\hline & +3 & $7(13.2)$ & $5(71.4)$ & $2(28.6)$ \\
\hline & +4 & $22(41.5)$ & $22(100)$ & $0(0.0)$ \\
\hline \multirow[t]{5}{*}{ Tracheal aspirate (10) } & Total TA & $10(100)$ & $4(40)$ & $6(60)$ \\
\hline & AFB -ve & $4(40)$ & $0(0.0)$ & $4(100)$ \\
\hline & $A F B+v e$ & $6(60)$ & $4(66.6)$ & 2(33.3) \\
\hline & +1 & $5(50)$ & $3(60)$ & $2(40)$ \\
\hline & +3 & $1(10)$ & $1(100)$ & $0(0.0)$ \\
\hline \multirow[t]{6}{*}{ Bronchial wash (11) } & Total BW & $11(100)$ & $8(72.7)$ & $3(27.3)$ \\
\hline & AFB -ve & $3(27.3)$ & $0(0.0)$ & $3(100)$ \\
\hline & $A F B+v e$ & $8(72.7)$ & $8(100)$ & $0(0.0)$ \\
\hline & +1 & $5(45.5)$ & $5(100)$ & $0(0.0)$ \\
\hline & +2 & $2(18.2)$ & $2(100)$ & $0(0.0)$ \\
\hline & +3 & $1(9.1)$ & $1(100)$ & $0(0.0)$ \\
\hline CSF (5) & Total CSF - ve & $5(100)$ & $3(60)$ & $2(40)$ \\
\hline \multirow[t]{4}{*}{ Cyst (7) } & Total Cyst & $7(100 \%)$ & $6(85.7)$ & $1(14.3)$ \\
\hline & - ve & $3(42.9)$ & $2(66.7)$ & $1(33.3)$ \\
\hline & +1 & $1(14.3)$ & $1(100)$ & $0(0.0)$ \\
\hline & +4 & $3(42.9)$ & $3(100)$ & $0(0.0)$ \\
\hline \multirow[t]{3}{*}{ Tissue (11) } & Total Cyst & $11(100)$ & $4(36.4)$ & $7(63.6)$ \\
\hline & - ve & $8(72.7)$ & $1(12.5)$ & $7(87.5)$ \\
\hline & +1 & $3(27.3)$ & $3(100)$ & $0(0.0)$ \\
\hline \multirow[t]{3}{*}{ Gastric aspirate (2) } & Total GA & $2(100)$ & $0(0.0)$ & $2(100)$ \\
\hline & - ve & $1(50)$ & $0(0.0)$ & $1(100)$ \\
\hline & +2 & $1(50)$ & $0(0.0)$ & $1(100)$ \\
\hline \multirow[t]{3}{*}{ Peritoneal fluid (2) } & Total PTF & $2(100)$ & $1(50)$ & $1(50)$ \\
\hline & -ve & $1(50)$ & $0(0.0)$ & $1(100)$ \\
\hline & +1 & $1(50)$ & $1(100)$ & $0(0.0)$ \\
\hline
\end{tabular}


Table 3. MTBC detection of AFB-negative, AFB-positive, pulmonary and extra-pulmonary specimens by Gene Xpert

\begin{tabular}{|c|c|c|}
\hline \multirow{2}{*}{ AFB staining results (no.) } & \multicolumn{2}{|c|}{ Detection of MTBC by PCR } \\
\hline & No.(\%)Detected & No. (\%)Not detected \\
\hline AFB-negative (34) & $10(29.4)$ & $24(70.6)$ \\
\hline AFB-positive (67) & $59(88.1)$ & $8(11.9)$ \\
\hline$A F B+1(25)$ & $20(80)$ & $5(20)$ \\
\hline $\mathrm{AFB}+2(8)$ & 7 (87.5) & $1(12.5)$ \\
\hline$A F B+3(9)$ & $7(77.8)$ & $2(22.2)$ \\
\hline$A F B+4(25)$ & $25(100)$ & $0(0.0)$ \\
\hline Total pulmonary specimens (74) & $55(74.3)$ & $19(25.7)$ \\
\hline AFB-ve pulmonary specimens (16) & $4(25)$ & $12(75)$ \\
\hline AFB +ve pulmonary specimens (58) & $51(87.9)$ & $7(12.1)$ \\
\hline Total extra-pulmonary specimens (27) & 14(51.9) & 13(49.1) \\
\hline AFB-ve extra-pulmonary specimens (18) & $6(33.3)$ & $12(66.7)$ \\
\hline AFB +ve extra-pulmonary specimens (9) & 8 (88.8\%) & $1(11.2)$ \\
\hline Total samples (101) & $69(68.3)$ & $32(31.7)$ \\
\hline
\end{tabular}

traditional costumes of females in Saudi Arabia with covering their faces by (Hijab) may reduce the incidence of exposure to droplet infections. Gender distribution was similar to the studies of Erbes et al. [12], and the study of Al shimemeri et al. [1] has indicated that males were diagnosed more often with severe pulmonary tuberculosis than females.

The present study found that AFB-positive sputum samples were representing $83 \%$ of total sputum samples, and were divided into $18.9 \%(+1)$, $9.4 \%(+2)$ and $13.2 \%(+3)$ (Table 2). While Khatib et al. [13] discovered that (28.6\%) of the sputum samples were +2 followed by $(23.8 \%)$ as +1 and $(17.1 \%)$ as +3 .
The sensitivity of PCR MTBC detection was 29.4\% and $88.1 \%$ in positive and negative AFB samples, respectively (Table 3 ). Our results were less than those of loannidis et al. [14] and Mishra et al. [15] who reported that the sensitivity of PCR for MTBC detection of AFB-positive samples were 100\% and 96.4\%, respectively. Our findings regarding $P C R$ detection of MTBC in AFB-negative samples did not correlate well with those reported by others [16,17], since their studies were performed mainly on respiratory samples. Also, the higher sensitivity for AFB-negative specimens in those studies were obtained following using more than one specimen for each patient. The sensitivity of PCR for MTBC detection of total pulmonary, AFBpositive and AFB-negative pulmonary samples were 
Table 4. Detection time of MTBC by VersaTREK according to AFB staining results.

\begin{tabular}{|c|c|c|}
\hline Types of specimen (No.) & AFB staining (No.) * & $\begin{array}{l}\text { Detection time (days) by Versa TREK } \\
\text { (Mean } \pm \text { SD) }\end{array}$ \\
\hline \multirow{5}{*}{ Sputum (53) } & - ve(9) & $26.66 \pm 6.32$ \\
\hline & $+1(10)$ & $21.33 \pm 4.84$ \\
\hline & $+2(5)$ & $14.6 .33 \pm 4.39$ \\
\hline & $+3(7)$ & $15.8 \pm 7.9$ \\
\hline & $+4(22)$ & $11.52 \pm 5.61$ \\
\hline \multirow{3}{*}{ Tracheal aspirate (10) } & $-\operatorname{ve}(4)$ & $27.75 \pm 7.93$ \\
\hline & $+1(5)$ & $21.61 \pm 5.77$ \\
\hline & $+3(1)$ & 7 \\
\hline \multirow{4}{*}{ Bronchial wash (11) } & - ve(3) & $27.33 \pm 11.71$ \\
\hline & $+1(5)$ & $20.66 \pm 8.36$ \\
\hline & $+2(2)$ & $10.5 \pm 0.701$ \\
\hline & $+3(1)$ & 7 \\
\hline Cerebrospinal fluid (5) & - ve(5) & $23 \pm 2.7$ \\
\hline \multirow{3}{*}{ Cyst (7) } & - ve(3) & $25.66 \pm 6.65$ \\
\hline & $+1(1)$ & 21 \\
\hline & $+4(3)$ & $13 \pm 1.41$ \\
\hline \multirow{2}{*}{ Tissue (11) } & - ve(8) & $28.28 \pm 8.67$ \\
\hline & $+2(3)$ & $24.33 \pm 10.4$ \\
\hline \multirow{2}{*}{ Gastric aspirate (2) } & - ve(1) & 26 \\
\hline & $+2(1)$ & 15 \\
\hline \multirow{2}{*}{ Peritoneal fluid (2) } & $-v e(1)$ & 30 \\
\hline & $+1(1)$ & 24 \\
\hline Total AFB (67) & + ve & $15.01 \pm 7.32$ \\
\hline Total AFB (34) & -ve & $26.63 \pm 6.7$ \\
\hline
\end{tabular}

*-ve= negative $;+\mathrm{ve}=$ positive . 
Table 5. Anti-tuberculous drug resistance patterns of MTBC in Saudi and non-Saudi TB patients.

\begin{tabular}{|c|c|c|c|c|}
\hline Pattern of Anti-TB drugresistance & $\begin{array}{l}\text { Total no.(\%) } \\
\text { resistance }\end{array}$ & Saudis*No. (\%) & Non-Saudis* No. (\%) & P-value \\
\hline Any resistance to RIF & $4(3.96)$ & $3(75)$ & $1(25)$ & 0.206 \\
\hline Anyresistance to INH & $4(3.96)$ & $2(50)$ & $2(50)$ & 0.57 \\
\hline Anyresistance to PZA & $14(13.86)$ & $6(42.9)$ & 8(59.1) & 0.61 \\
\hline Anyresistance to Streptomycin & $8(7.9)$ & $2(25)$ & $6(75)$ & 0.25 \\
\hline MDR (RIF+INH) & $2(1.98)$ & $1(50)$ & $1(50)$ & 0.62 \\
\hline Mono resistance to RIF & $2(1.98)$ & $2(100)$ & $0(0.0)$ & 0.17 \\
\hline Mono resistance to INH & $1(0.99)$ & $0(0.0)$ & $1(100)$ & 0.57 \\
\hline Mono resistance to PZA & $12(11.88)$ & $4(33.3)$ & $8(66.7)$ & 0.49 \\
\hline Mono resistance to Streptomycin & $7(6.93)$ & $1(14.3)$ & $6(85.7)$ & 0.11 \\
\hline Any drug resistance & $25(24.75)$ & $9(36)$ & $16(64)$ & 0.29 \\
\hline One drug resistance & $22(21.78)$ & $7(31.8)$ & $15(68.2)$ & 0.24 \\
\hline Two drugs resistance & $3(2.97)$ & $2(66.7)$ & $1(33.3)$ & 0.47 \\
\hline Four drugs resistance & $1(0.99)$ & $1(100)$ & $0(0.0)$ & 0.24 \\
\hline No drug resistance & $76(75.25)$ & $34(44.7)$ & $42(55.3)$ & 0.29 \\
\hline
\end{tabular}

*No of Saudi patients (43) and non-Saudi patients (58).

74.3\%, 87.9\% and 25\%, respectively (Table 3). Zeka et al. [18] reported that the sensitivity of PCR for pulmonary specimens were $74.2 \%$ and $100 \%$ for both of negative and positive specimens, respectively. While Omrani et al. [19] reported also that, 23.5\% of their AFB-negative pulmonary TB samples were detected by PCRin their study in the Prince Sultan Military Medical City, Riyadh, KSA .

This study demonstrates that the sensitivity of PCRfor MTBC detection of total pulmonary, AFBpositive and AFB-negative extra-pulmonary samples were $51.9 \%, 88.8 \%$ and $33.3 \%$, respectively (Table 3), while the study of Zeka et al. [18] reported sensitivity of $63 \%$ and $100 \%$ for negative and positive specimens, respectively. Omrani et al. [19] reported also that $24.4 \%$ of AFB-negative extrapulmonary TB samples included in their study were detected by PCR.

In the current study, the detection time of MBTC by VersaTREK for AFB-positive samples $(15.01 \pm 7.32$ days) was shorter than that reported by Falconi et al. [20] who observed that primary isolation of $M$. tuberculosis from smear-positive sputum samples was $19.8 \pm 11.2$ days. However, the detection time of smear-positive pulmonary samples was comparable to that of extra-pulmonary samples in our study (Table 4). Zeka et al. [18] found that the median time for smear-positive samples was statistically shorter for pulmonary specimens than in extra-pulmonary specimens. This finding could be due to low number of organisms in the extrapulmonary specimens.

The present study shows that $24.75 \%$ of $M$. tuberculosis isolates were found to be resistant to one or more of anti-TB drugs (Table 5). This percentage was higher than that reported by the WHO in 2006 and 2007 (10.2\%) [3]. However, other studies revealed that the rates of resistance of $M$. tuberculosis isolates in Saudi Arabia were similarly high as demonstrated in our study [21,22]. Alrajhi 
and Al-Barrak [21] showed in their meta-analysis of previous local studies which based on 12 reports from several regions of the Kingdom a wide range of resistance from 8.7 to $43.7 \%$. Lower rates of resistance have also been reported from two other countries in the region (Qatar and Oman) as well as from countries with low TB incidence such as Western Europe and North America [3]. Other Middle East Arab countries such as Lebanon and Egypt, reported higher levels of drug-resistant TB and MDR-TB $[22,23]$. Much higher resistance rates were also reported from TB-endemic countries of South Asia and South-East Asia [3].

This study shows that the PZA resistant strains (13.86\%) among $M$. tuberculosis isolates was high. Following further identification of these strains using Hain PCR method revealed that 12 out of 14 PZA resistant strains were $M$. canetti which are known to be intrinsic resistant to PZA, and $M$. canetti is a novel pathogenic taxon of the $M$. tubcerculosis. The next highest resistance was observed with streptomycin (7.9\%) followed by similar rates of resistance to both rifampicin and $\mathrm{INH}$ (3.96\%) (Table 5).

In KSA, the resistance rates to isoniazid in Riyadh, ranged from 4.2 to $7.2 \%$ [24,25]. Similar resistance rates of about $6 \%$ were reported in both Dammam [25] and in Taif [26]. A higher resistance rates (10.3 to $28.7 \%$ ) were reported in Jeddah $[\mathbf{2 7}, \mathbf{2 8}]$, and the highest rate of resistance (41\%) was reported from Jazan and was attributed to the proximity ofJazan to the Republic of Yemen [29]. Additionally, rifampicin-resistance rate was low $(3.96 \%)$, and is similar to the low rifampicinresistance rate reported from Dammam [25], where as higher rates of rifampicin-resistance were reported from Riyadh (9\%) [24], Jeddah (5.1 to 23.4\%) [4,27] and Taif (15.3\%) [26].

This study shows that streptomycin-resistance of $M$. tuberculosis was $7.9 \%$, while the study from Riyadh, found $8.8 \%$ streptomycin-resistance [30], and higher resistance rates were reported from Taif (15.9\%) [26] and Jeddah (22.7\%) [29].
Ethambutol-resistance has also been reported to be variable in Saudi Arabia, but it was null in our study. The same result was reported in Dammam [25], while other studies demonstrated higher results of resistance (1.3 to 6.9\%) in Jeddah [4,27] and each 4\% in both Taif [26] and Jazan [30].

According to our DST results, two isolates (1.98\%) were detected as MDR strains (Table 5). The rate of MDR-TB in Saudi Arabia is relatively low and variable between Saudi provinces. The first representative national survey conducted in Saudi Arabia by Al-Hajoj et al. [31], and which included all patients diagnosed with culture-positive TB, found that the highest proportion was detected in Northern provinces (9.19\%) and the second highest proportion was found in Southern provinces (5.4\%), followed by Western provinces (4.9\%) and Central provinces (3.8\%). The lowest proportion of MDRTB isolates was reported from the Eastern province $(1.1 \%)$. On the other hand, a very high rate of MDRTB was reported from Jazan, where the resistance rate reached $44 \%$ [30].

The variability in the resistance rates of tuberculosis in Saudi Arabia is dependent on the time and location of the study as well as on the numbers of non-Saudi patients in each study [32]. In our study, the rates of resistance to pyrazinamide and streptomycin were higher among non-Saudis than in Saudis with no significant difference. Also, the rates of mono-resistance to INH, PZA and streptomycin were higher in non-Saudis than in Saudis with no significant difference (Table 5). These results were compatible with that obtained by Al-Tawfiq et al. [33] during their 15-year study (1989-2003) in Saudi Aramco Medical Services Organization in Dhahran. Our result matched also with that of Varghese et al.[34] who reported no significant differences of drug- resistance rate between immigrants and local population in Saudi Arabia. Therefore, screening of expatriate workers and illegal immigrants into the Kingdom might help to control spread of TB infection. The Ministry of Health of the KSA has 
implemented such a plan and its effects will be evaluated over the next few years [34].

In conclusion, this study found that the rate of MTBC detected by PCR was higher in positive than negative $A F B$, and generally low rates of resistance were found against all anti-TB drugs. There was no significant difference between Saudi and nonSaudi TB patients regarding any type of resistance to anti-TB drugs. Further studies on incidence of TB and continued surveillance of resistance pattern of $M$. tuberculosis isolates are needed to formulate effective plans for the future management of TB.

\section{Conflict of interest}

This study was not supported or funded by any drug company. The authors declare that there is no conflict of interests regarding the publication of this paper.

\section{References}

1. Alshimemeri AA, Arabi YM, Al-Jahdali H, Olayan A, Al Harbi O, Memish Z. Clinical presentation and outcome of patients diagnosed with active pulmonary tuberculosis in a large critical care unit. Crit Care \& Shock 2011; 14: 1-6.

2. Al- Jasser FS, Kabbash IA, Almazroa MA, Memish ZA. Patterns of diseases and preventive measures among domestic hajjis from Central, Saudi Arabia. Saudi Med J 2012; 33: 879-86.

3. World Health Organization (2007) Global tuberculosis control: surveillance, planning, financing. WHO/HTM/TB/2007.376. Geneva, Switzerland.

4. Zaman R. Tuberculosis in Saudi Arabia: initial and secondary drug resistance among indigenous and non-indigenous populations. Tubercle.1991; 72: 51-55.

5. Mandourah Y, Al-Radi A, Ocheltree AH, Ocheltree SR, Fowler RA. Clinical and temporal patterns of severe pneumonia causing critical illness during Hajj. BMC Infect Dis 2012;112:17.

6. World Health Organization (WHO) (2008)(4) Molecular line probe assays for rapid screening of patients at risk of multidrugresistant tuberculosis (MDR-TB): policy statement. WHO, Geneva, Switzerland.

7. Centers for Disease Control and Prevention (CDC) (1995) Mycobacterium tuberculosis: Assessing your Laboratory.

8. Boehme CC, Nabeta P, Hillemann D, NicolMP, Shenai S, Krapp F, et al. Rapid Molecular Detection of Tuberculosis and Rifampin
Resistance. N Engl J Med 2010; 363: 1005-15.

9. MB/BacT Detection system manual; http://www.biomerieux. com,2008

10. Espinal, M. A.. The global situation of MDR-TB. Tuberculosis (Edinburgh) 2003; 83:44-51.

11. Abouzeid MS, Zumla Al, Felemban S, Alotaibi B, O'Grady J, Memish ZA. Tuberculosis Trends in Saudis and Non-Saudis in the Kingdom of Saudi Arabia: A 10 Year Retrospective Study (2000-2009). PLoS ONE 2012; 7 (6): e39478.

12. Erbes R, Oettel K, Raffenberg M, Mauch $H$, Schmidt-loanas $M$, et al. Characteristics and outcome of patients with active pulmonary tuberculosis requiring intensive care. Eur Respir J 2006; 27:1223-28.

13. Khatib SI, Williamson MT, Singh R, Joshi JM. Diagnosis of pulmonary tuberculosis by smear microscopy and culture in a tertiary health care facility. Biology and Medicine 2012; 1(4):3236.

14. Ioannidis $P$, Papaventsis $D$, Karabela S, Nikolaou S, Panagi M, et al. Cepheid GeneXpert MTB/RIF Assay for Mycobacterium tuberculosis Detection and Rifampin Resistance Identification in Patients with Substantial Clinical Indications of Tuberculosis and Smear-Negative Microscopy Results. J Clin Microbiol 2011;49 (8):3068-70.

15. Mishra, PK, Gorantla VR, Bhargava A, Varshney S, Vashistha $P$, et al. Molecular detection of Mycobacterium tuberculosis in formalin-fixed, paraffin-embedded tissues and biopsies of gastrointestinal specimens using real-time polymerase chain reaction system. Turk J Gastroenterol. 2010; 21(2):129-34.

16. Hillemann D, Rusch-Gerdes S, Boehme C. Richter E . Rapid molecular detection of extrapulmonary tuberculosis by the automated GeneXpert MTB/RIF system. J Clin Microbiol 2011; 49: 1202-1205.

17. Moure R, Munoz L, Torres M, Santin R, Alcaide F. Rapid detection of Mycobacterium tuberculosis complex and rifampin resistance in smear-negative clinical samples by use of an integrated realtime PCR method. J Clin Microbiol 2011;49:1137-39.

18. Zeka AN, Tasbakan S and Cavusoglu C. Evaluation of the GeneXpert MTB/RIF Assay for Rapid Diagnosis of Tuberculosis and Detection of Rifampin Resistance in Pulmonary and Extrapulmonary Specimens. J Clin Microbiol 2011;49(12): 413841.

19. Omrani AS, Al-Otaibi MF, Al-Ateah SM, Al-Onazi FM, Baig K, et al. GeneXpert MTB/RIF Testing in the Management of Patients with Active Tuberculosis; A Real Life Experience from Saudi Arabia. Infect Chemother. 2014; 46(1):30-34.

20. Falconi FQ, Suárez LI, LópezMde J, Sancho CG. Comparison of the VersaTrek system and Löwenstein-Jensen medium for the recovery of mycobacteria from clinical specimens. Scand J Infect Dis 2008;40: 49-53.

21. Alrajhi AA and Al-Barrak AM. Mycobacterium tuberculosis susceptibility in Saudi Arabia. Saudi Med J 2002;23: 1227-31.

22. Kordy FNS, Al-Thawadi S, Alrajhi AA. Drug resistance patterns of Mycobacterium tuberculosis in Riyadh, Saudi Arabia. Int J Tuberc Lung Dis 2004;8:1007-11.

23. Araj GF, Itani L, Kanj NA. Comparative study of antituberculous drug resistance among Mycobacterium tuberculosis isolates 
recovered at the American University of Beirut Medical Center: 1996-1998 vs. 1994-1998. Leb Med J. 2000; 48: 18-22.

24. Al- Orainey IO. Resistance to antituberculosis drugs in Riyadh, Saudi Arabia. Tubercle. 1989;70: 207-10.

25. Al- RubaishA M, Madania AA, Al-Muhanna FA. Drug resistance pulmonary tuberculosis in the Eastern Province of Saudi Arabia Saudi Med J. 2001;22: 776-79.

26. Jarallah JS, Elias AK, al Hajjaj MS, Bukhari MS, al Shareef $\mathrm{AH}$, al-Shammari SA. High rate of rifampicin resistance of Mycobacterium tuberculosis in the Taif region of Saudi Arabia. Tuber Lung Dis 1992;73: 113-15.

27. Kinsara AJ. Review of non-tuberculous mycobacteria: King Khalid National Guard Hospital, Jeddah, Saudi Arabia. Saudi Med J. 1997;19: 212-14.

28. Khan MY, Kinsara AGand Osoba AO. Increasing resistance of M tuberculosis to anti-TB drugs in Saudi Arabia. Int J Antimicrob Agents Chemother. 2001;17: 415-18.

29. Schiott CR, Engback HC, Vergmann B, Al-Motez M, Kassim I. Incidence of drug resistance amongst isolates of Mycobacterium tuberculosis recovered in Gizan area, Saudi Arabia. Saudi Med J 1985;6:375-78.

30. Singla R, Al-Sharif N, Al-Sayegh M, Osman M and Shaikh MA. Prevalence of resistance to anti-tuberculosis drugs in Riyadh and a review of previous reports. Ann Saudi Med 2003;23: 143-47.

31. Al- Hajoj S, Varghese B, Shoukri MM, Al-Omari R, Al-Herbwai,AlRabiah F et al. Epidemiology of Antituberculosis Drug Resistance in Saudi Arabia: Findings of the First National Survey. Antimicrob Agents Chemoth 2013; 57 (5): 2161-66.

32. Abu-Amero KK. Status of antituberculosis drug resistance in Saudi Arabia 1979-98. East MedHealth J 2002; 8: 664- 670.

33. Al- Tawfiq JA, Al-Muraikhy AA, Abed MS. Susceptibility Pattern and Epidemiology of Mycobacterium tuberculosis in a Saudi Arabian Hospital A 15-Year Study From 1989 to 2003. CHEST 2005; 128: 3229-32.

34. Varghese B, Supply $P$, Shoukri M, Allix-Beguec C, Memish $\mathrm{Z}$, Abuljadayel $\mathrm{N}$, et al.Tuberculosis Transmission among Immigrants and Autochthonous Populations of the Eastern Province of Saudi Arabia. PLoS ONE 2013; 8(10): e77635.

\section{Comment on this article:}

\section{(f) (B) in $8+S$. $P$}

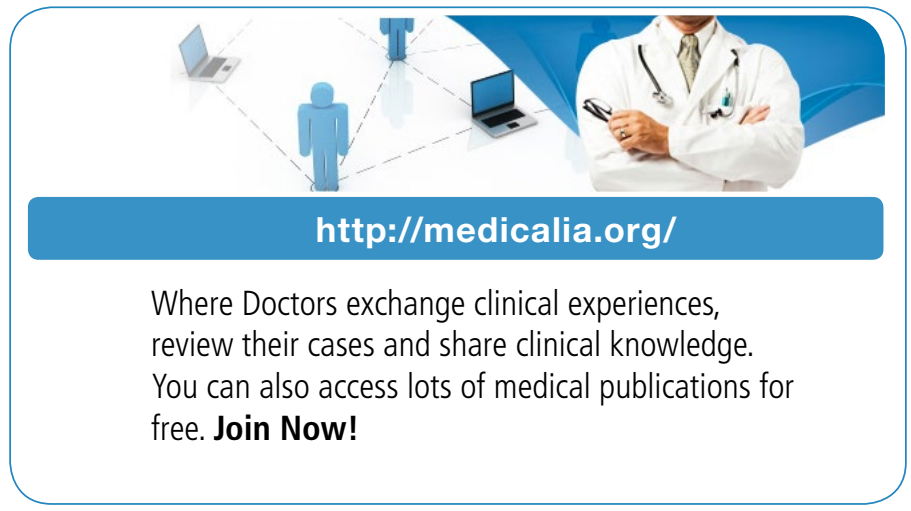

\section{Publish with iMedPub}

http://www.imed.pub

The Journal is an open access peer-reviewed journal that publishes scientific papers about all aspects of antimicrobials. The journal will publish original research articles, reviews, brief reports and case reports dealing with basic and clinical antibacterial agents, antiviral, antiprotozoals, antituberculuous, antifungal and antihelminthes agents.

All manuscripts must be prepared in English, and are subject to a rigorous and fair peer-review process. Accepted papers will immediately appear online.

The journal aims to advance the knowledge, attitude and the research of chemotherapy in the Arabic world in cooperation with international, national scientific and public societies as well as research centers with similar aims and objectives. 